\title{
Modeling induced resistance to plant disease using a dynamical systems approach
}

\author{
Nurul S. Abdul Latif ${ }^{1,2}$, Graeme C. Wake ${ }^{2}$, Tony Reglinski ${ }^{3}$, Philip A. G. Elmer $^{3}$ and Joseph T. Taylor ${ }^{3}$ \\ ${ }^{1}$ Faculty of Agro Based Industry, Universiti Malaysia Kelantan, Jeli, Kelantan, Malaysia \\ 2 Institute of Information and Mathematical Sciences, Massey University, Auckland, New Zealand \\ ${ }^{3}$ The New Zealand Institute for Plant and Food Research Limited, Hamilton, New Zealand \\ *Correspondence: tony.reglinski@plantandfood.co.nz
}

Edited by:

Saskia C. M. van Wees, Utrecht University, Netherlands

Reviewed by:

Michael Jeger, Imperial College London, UK

Standard epidemiological models describe that Susceptible plants $(S)$ will become infected and develop Disease $(D)$ after inoculation with a compatible pathogen under appropriate environmental conditions. These dynamic relationships can be affected by subtle changes to any one parameter and may result in a proportion of the plant population being able to exhibit Resistance $(R)$ to infection. An example of this is the use of elicitors to promote an increase in plant basal resistance and so enable a proportion of formerly susceptible plants to express resistance i.e., a shift in the population from $S$ to $R$. This phenomenon is termed induced resistance (IR). In this paper, a prototype mathematical model of IR is presented to describe the effects of a chemical elicitor compound, methyl jasmonate (MeJA), on the resistance of Pinus radiata seedlings to Diplodia pinea the causal agent of pine stem canker and tip dieback. Pine seedlings were sprayed with $0.1 \%$ MeJA at 27, 13, 6, or 3 days before inoculation with $D$. pinea using methods previously described by Gould et al. (2008). Disease assessments commenced at 1 week after inoculation and continued at 3-4 day intervals thereafter for 5 weeks. Disease development on the MeJA-treated seedlings was compared to that on a cohort of untreated plants. In this model system, the IR response is transient and it is modeled here using a forward-and-backward kinetics framework to describe the temporal nature of the phenomenon.

Because the expression of IR can only be detected after pathogen challenge, the model is formulated with the treated plants divided into two regimes: (1) preinoculation and (2) post-inoculation. The assumptions for the model's formulation can be summarized as follows. The plant population is divided into three compartments according to the above definitions where $S+R+D=1$. At the time when plants are treated with an elicitor $(t=0)$, a proportion of the plant population will exhibit natural resistance $\left(R_{i}\right)$. The induction period $\left(t_{p}\right)$ describes the time interval between elicitor application and pathogen inoculation. Upon inoculation, a proportion of plants $\left(D_{i}\right)$ will become infected immediately. This prototype IR model is based on the model by Jeger et al. (2009) and $\mathrm{Xu}$ et al. (2010). The model's equations for the treated plants are as follows:

Pre-inoculation: For $0 \leq t<t_{p}$

$$
\begin{aligned}
\frac{d R}{d t} & =(e(t)-\gamma R)(1-R) ; \\
R(0) & =R_{i}
\end{aligned}
$$

Post-inoculation: For $t_{p} \leq t \leq T$

$$
\begin{aligned}
\frac{d R}{d t} & =(e(t)-\gamma R)(1-R-D) ; \\
R\left(t_{p}\right) & =R_{p} \\
\frac{d D}{d t} & =\beta D(1-R-D) ; \\
D\left(t_{p}\right) & =D_{i}
\end{aligned}
$$

where we take $e(t)=\frac{k t}{t^{2}+L^{2}}\left[\right.$ days $\left.^{-1}\right]$ as the elicitor effectiveness in the plants where $\frac{k}{2 L}\left[\right.$ days $\left.^{-1}\right]$ is the maximum elicitor effect and $L$ [days] is the time where this is at its peak, $\gamma\left[\right.$ days $\left.^{-1}\right]$ is the rate that resistant tissue becomes susceptible, and also $\beta\left[\right.$ days $^{-1}$ ] is the rate of disease development. The form for $e(t)$ is chosen to reflect the temporal nature of IR with an initial increase in resistance which then decays over time. $R_{p}$ is the degree of the resistance at the time of the pathogen inoculation obtained from Equation (1). Also, $T$ is a finite (sufficiently large) time after the pathogen inoculation. The Equations (1)-(3) are based on the assumption that the rate of change of $R$ and $D$ are directly proportional to the amount of $S$ available at a particular time (that is, $S=1-R-D$ ). For Equation (1), $D$ is not in the equation because the pathogen is absent during this period. Pathogen is inoculated at time $t_{p}$, therefore the $D$ term is occurs only in Equations (2) and (3). For the untreated plants, they share the same parameter values, especially $\beta, R_{i}, D_{i}$, since these untreated plants are characterized as the control group. The untreated plants will not have the $e(t)$ term in their model equations and so are autonomous, that is, independent of time except implicitly. Therefore, the model's equations for the untreated plants are as follows:

$$
\begin{aligned}
\frac{d R}{d t} & =\alpha R(1-R-D) ; \\
R(0) & =R_{i} \\
\frac{d D}{d t} & =\beta D(1-R-D) ; \\
D(0) & =D_{i}
\end{aligned}
$$

where $\alpha\left[\right.$ days $\left.^{-1}\right]$ is the rate that untreated susceptible tissue becomes resistant.

The seven unknown parameters $\left(\alpha, \beta, \gamma, k, L, R_{i}, D_{i}\right) \quad$ are determined by matching data to the model using computer-based algorithms. The following figure (Figure 1) was plotted using the optimal parameter values, and it illustrates the characteristics of the two compartments $R$ and $D$ for the Equations (1)-(5). 


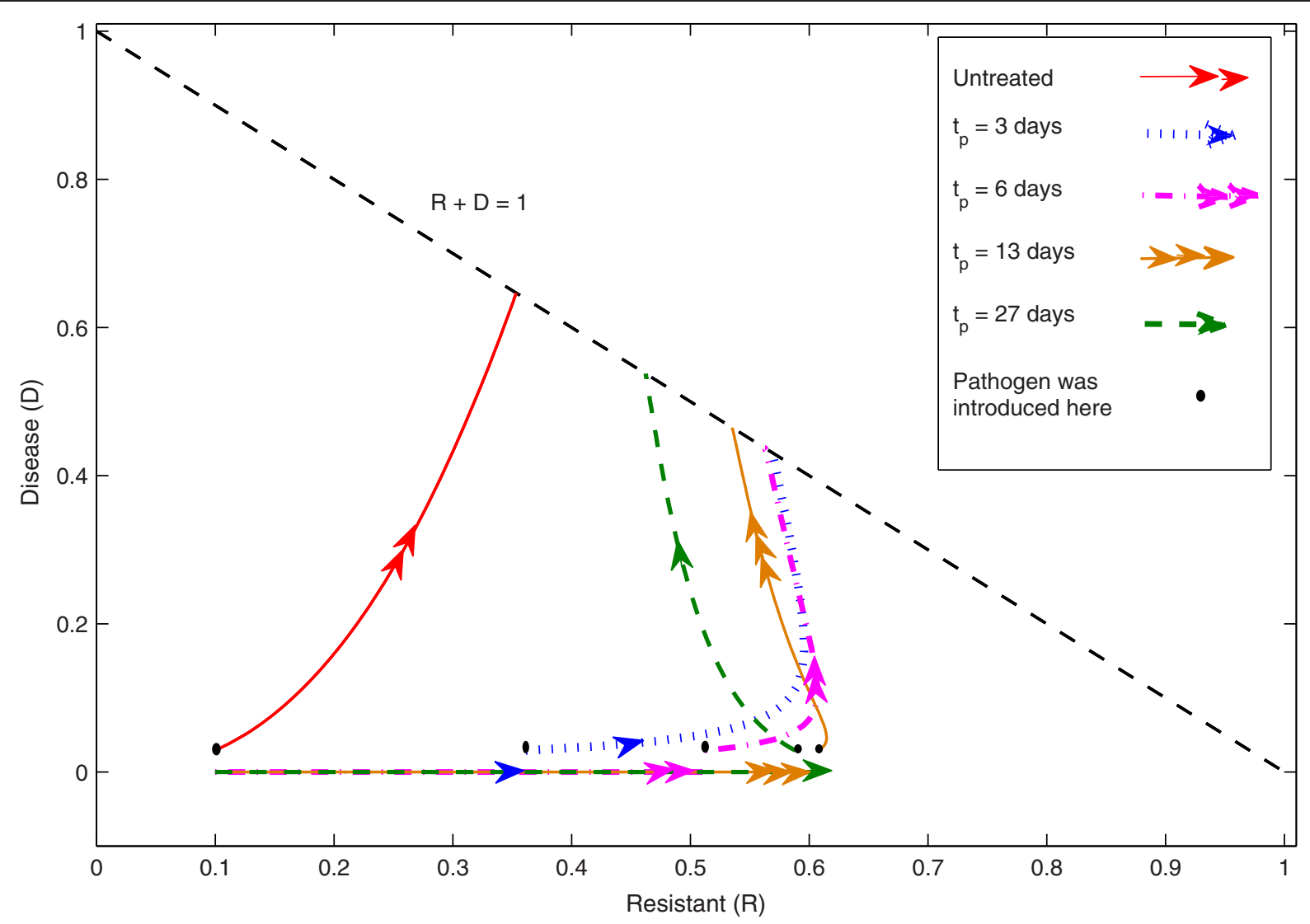

FIGURE 1 | The phase-plane for the induced resistance (IR) model. These trajectories of the $R, D$ compartments are plotted in the feasible region $(R, D>0, R+D<1)$. The lines schematically represent the values of the two compartments $R$ and $D$ as time passes for the Equations (1)-(5) based on each induction case $t_{p}$ and the untreated case. When $D=0$, the straight lines illustrate the dynamics of the $R$ compartment before the pathogen inoculation. These lines will have a discontinuity when the pathogen is introduced at time $t=t_{p}$, and it shows the state of the $R$ compartment at that particular time. As can be seen in the figure, there is a jump in the $D$ values of $D_{0}$ at $t=t_{p}$ and the trajectories of the $R, D$ compartments will continue to approach the straight line $R+D=1$. For the untreated case, the dynamics of the $R, D$ compartments will depend on the initial condition of the systems i.e., $R_{i}$ and $D_{i}$.
The flows in the figure indicate the timeevolution of the system of the differential equations described above based on the different values of $t_{p}$ and the untreated case. It shows that when $t_{p}$ is between 3 and 6 days, the subsequent development of disease is less severe than with the other induction times and with the untreated plants. That is, the resistance induced by the elicitor application is at its peak. The figure shows that the trajectories for each induction case will eventually approach that straight line $R+D=1$. The model has three compartments $(S, R, D)$, but the data sets have only two $([S+R], D)$. The Susceptible cohort is given by $S=1-R-D$ and can be separated out by this model. The model has a line of equilibrium states in the $R, D$ plane which are attracting, and predicts that disease development depends on the induction time $t_{p}$.

\section{CONCLUSION}

The management of plant diseases involves an assessment of the risks and the costs, both economic and environmental, associated with the implementation of different control measures. Various disease risk prediction models have been developed as decision support tools to facilitate more efficient use of management options; these are generally based on the rationale that pest and disease development follow predictable life cycles and that by monitoring key epidemiological parameters it is possible to target more accurately events critical for management (Gent et al., 2011). Disease risk prediction models may prove critical for coordination of elicitor application in crop production systems because of the importance of early intervention when relying on IR for disease control. In this study we discuss the development of a prototype mathematical model to predict the temporal dynamics of chemically-IR. The current model offers the potential to quantitatively estimate the effectiveness of elicitor treatment and to predict the relative proportion of plants exhibiting IR to pathogen inoculation. Moreover, the model is generic and will be applicable for a range of plant-pathogenelicitor scenarios. For future work, this prototype IR model will be extended to predict the required $t_{p}$ to achieve optimum disease control. In addition, it will be interesting to observe the dynamics of the system when there are multiple elicitor applications to plants, a scenario 
which may be needed in practice. This is important in practical terms because successful application of elicitors will require knowledge of the onset and duration of the IR response. This new model will complement and extend the value of risk prediction models by providing decision support on the timing and frequency of elicitor applications for management of disease.

\section{ACKNOWLEDGMENTS}

The authors wish to thank the New Zealand Ministry for Business, Innovation and Employment (LINX0804 "Ecosystem Bioprotection") and Ministry of Higher Education of Malaysia (KPTBS-851110105416) for funding to support this study.

\section{REFERENCES}

Gent, D. H., De Wolf, E., and Pethybridge, S. J. (2011). Perceptions of risk, risk aversion, and barriers to adoption of decision support systems and integrated pest management: an introduction. Phytopathology 101, 640-643.

Gould, N., Reglinski, T., Spiers, M., and Taylor, J. T. (2008). Physiological trade-offs associated with methyl jasmonate - induced resistance in Pinus radiata. Can. J. For. Res. 38, 677-684.

Jeger, M. J., Jefferies, P., Elad, Y., and Xu, X. M. (2009). A generic theoretical model for biological control of foliar plant disease. J. Theor. Biol. 256, 201-214.

Xu, X. M., Salama, N., Jeffries, P., and Jeger, M. J. (2010). Numerical studies of biocontrol efficacies of foliar plant pathogens in relation to the characteristics of a biocontrol agent. Am. Phytopathol. Soc. 100, 814-821.

Received: 19 December 2012; accepted: 25 January 2013; published online: 18 February 2013.

Citation: Abdul Latif NS, Wake GC, Reglinski T, Elmer PAG and Taylor JT (2013) Modeling induced resistance to plant disease using a dynamical systems approach. Front. Plant Sci. 4:19. doi: 10.3389/fpls.2013.00019

This article was submitted to Frontiers in Plant-Microbe Interaction, a specialty of Frontiers in Plant Science. Copyright (c) 2013 Abdul Latif, Wake, Reglinski, Elmer and Taylor. This is an open-access article distributed under the terms of the Creative Commons Attribution License, which permits use, distribution and reproduction in other forums, provided the original authors and source are credited and subject to any copyright notices concerning any third-party graphics etc. 